\title{
Mittelbare Diskriminierung und Kausalität
}

\author{
Robert Rebhahn/Christoph Kietaibl"
}

A. Einführung 373

I. Unmittelbare und mittelbare Diskriminierung ........................ 373

II. Meinungsstand zum Kausalitätserfordernis 376

B. Besteht bei mittelbarer Diskriminierung ein Kausalzusammenhang zwischen unzulässigem Merkmal und Schlechterstellung?

I. Mittelbare Diskriminierung aus dem Blickwinkel unterschiedlicher Kausalitätskonzepte....................... 378

II. Sonderfälle mittelbarer Diskriminierung.... 383
C. Vermutung einer - kausalen - unmittelbaren Diskriminierung?............ 384

D. Entwicklung zum „kausalitätsfreien“ Verbot mittelbarer Diskriminierung... 388

I. Verschärfung der Rechtfertigungsprüfung ......................... 388

II. Kausalzusammenhang zwischen unzulässigem Merkmal und Korrelation?.............................. 392

III. Umgekehrter Kausalverlauf? ......... 393

E. Schlussbemerkung.................. 395

\section{A. Einführung}

\section{Unmittelbare und mittelbare Diskriminierung}

Diskriminierungsverbote zielen im Wesentlichen darauf ab, eine Schlechterstellung „aufgrund“ oder „wegen“ bestimmter, im jeweiligen Kontext unzulässiger Merkmale zu verhindern. Kausalität im Allgemeinen fragt nach dem bzw. ist der (Ursachen-)Zusammenhang zwischen zwei realen Ereignissen, allenfalls auch Zuständen. Dies legt es nahe, dass Fragen der Kausalität bei der Entfaltung der Diskriminierungsverbote eine gewichtige Rolle spielen. Anschaulich belegt dies auch die deutsche Diskussion zum Tatbestandsmerkmal „wegen“ in Art. 3 Abs. 3 GG, deren zentrales Thema die Prüfung des Kausalzusammenhanges zwischen Differenzierung und unzulässigem Unterscheidungsmerkmal ist ${ }^{1}$

Allerdings ist ein Kausalzusammenhang zwischen unerwünschtem Differenzierungsmerkmal und Schlechterstellung nur bei einer der beiden herkömmlichen Ausprägungen der Diskriminierung, der unmittelbaren, unzweifelhaft. Bei der anderen Ausprägung, der mittelbaren Diskriminierung, erscheint das Verhältnis von Diskriminierung(sverbot) und Kausalität hingegen eher unklar. Daher soll hier - aufgrund

* Univ.Prof. Dr. Robert Rebhahn ist Universitätsprofessor, Ass. Prof. Dr. Christoph Kietaibl ist Assistenzprofessor am Institut für Arbeits- und Sozialrecht der Universität Wien.

1 Vgl. dazu nur W. Heun, in: H. Dreier (Hrsg.), Grundgesetz-Kommentar, Bd. 1, 2. Aufl., Tübingen 2004, Art. 3 Rn. 119 ff.; G. Dürig/R. Scholz, in: T. Maunz/G. Dürig (Hrsg.), Grundgesetz, Teil B, 53. Aufl., München 2009, Art. 3 Rn. 133 ff.; F. Temming, Altersdiskriminierung im Arbeitsleben, München 2008, S. 103 ff.; A. Somek, Rationalität und Diskriminierung: zur Bindung der Gesetzgebung an das Gleichheitsrecht, Wien: Springer 2001, S. 435 f. 
einer Anregung durch die Herausgeber - der Bedeutung der Kausalität spezifisch für das Verbot mittelbarer Diskriminierung nachgegangen werden. ${ }^{2}$

Die praktisch wichtigsten Diskriminierungsverbote finden sich heute im Unionsrecht oder gehen doch darauf zurück. $\mathrm{Zu}$ nennen sind im Primärrecht insbesondere Art. 18 AEUV (Staatsangehörigkeit), die Bestimmungen zu den vier Freiheiten bzw. Freizügigkeiten (Art. 45, 49, 56 und 63 AEUV), Art. 157 Abs. 1 AEUV (Entgeltgleichheit) sowie nun auch Art. 21 GRC. Dazu treten Sekundärrechtsakte (Richtlinien) aufgrund der Ermächtigungen in Art. 19 und Art. 157 Abs. 3 AEUV, die sich insbesondere gegen Diskriminierungen im Arbeitsleben und bei der Versorgung der Öffentlichkeit richten, und die (auch) hier Gleichbehandlungs-Richtlinien genannt werden sollen. ${ }^{3}$ Sie verbieten Diskriminierungen aufgrund insbesondere des Geschlechts, der ethnischen Herkunft oder des Alters.

Bei der Diskriminierung wird heute weithin zwischen unmittelbarer und mittelbarer Diskriminierung unterschieden. ${ }^{4}$ Das Unionsrecht verwendet den Begriff „mittelbare Diskriminierung" allerdings nur im Sekundärrecht und hier fast nur in den Gleichbehandlungs-Richtlinien. ${ }^{5}$ Der Gemeinschaftsgesetzgeber hat den genannten Begriff zwar bereits 1976 erstmals verwendet, ${ }^{6}$ definiert hat er ihn aber erst 1997 in Art. 2 Abs. 2 Beweislast-RL. ${ }^{7}$ Eine mittelbare Diskriminierung lag danach vor, „wenn dem Anschein nach neutrale Vorschriften, Kriterien oder Verfahren einen wesentlich höheren Anteil der Angehörigen eines Geschlechts benachteiligen, es sei denn, die betreffenden Vorschriften, Kriterien oder Verfahren sind angemessen und notwendig und sind durch nicht auf das Geschlecht bezogene sachliche Gründe gerechtfertigt.“

2 Nicht eingegangen wird daher auf Fragen der Kausalität bei den Rechtsfolgen, z.B. bei der Frage, für welchen Zeitraum bei diskriminierender Nicht-Einstellung Entschädigung/Schadenersatz zu leisten ist. Diese Fragen stellen sich bei unmittelbarer Diskriminierung nämlich in gleicher Weise.

3 Insbes. Richtlinie 2006/54/EG zur Verwirklichung des Grundsatzes der Chancengleichheit und Gleichbehandlung von Männern und Frauen in Arbeits- und Beschäftigungsfragen (Neufassung); Richtlinie 2004/113/EG zur Verwirklichung des Grundsatzes der Gleichbehandlung von Männern und Frauen beim Zugang zu und bei der Versorgung mit Gütern und Dienstleistungen; Richtlinie 2000/78/EG zur Festlegung eines allgemeinen Rahmens für die Verwirklichung der Gleichbehandlung in Beschäftigung und Beruf; Richtlinie 2000/43/EG zur Anwendung des Gleichbehandlungsgrundsatzes ohne Unterschied der Rasse oder der ethnischen Herkunft.

4 Verschiedentlich wird im Schrifttum anstelle von mittelbarer Diskriminierung auch von faktischer Diskriminierung gesprochen (z.B. U. Kischel, in: V. Epping/C. Hillgruber (Hrsg.), Grundgesetz-Kommentar, 7. Aufl., München 2010, Art. 3 Rn. 165). Auch der EuGH tut dies manchmal (z.B. Rs. C-33/89, Kowalska, Slg. 1990, I-2591, Rn. 13; C-184/89, Nimz, Slg. 1991, I-297, Rn. 12). Der Unionsgesetzgeber verwendet aber zu Recht den treffenderen Begriff der mittelbaren Diskriminierung; vgl. zur Terminologie auch die Anmerkungen in Fn. 52.

5 Außerhalb der Gleichbehandlungs-Richtlinien verwendet der Unionsgesetzgeber den Begriff „,mittelbare Diskriminierung “ bisher nur in den Verordnungen 1072/2009 und 1073/2009 über den Zugang zum Markt des grenzüberschreitenden Güterkraftverkehrs und Personenkraftverkehrs, und auch dort nur in den Erwägungsgründen.

6 In Art. 2 der Richtlinie 76/207/EWG zur Verwirklichung des Grundsatzes der Gleichbehandlung von Männern und Frauen hinsichtlich des Zugangs zur Beschäftigung, zur Berufsbildung und zum beruflichen Aufstieg sowie in Bezug auf die Arbeitsbedingungen.

7 Richtlinie 97/80/EG über die Beweislast bei Diskriminierung aufgrund des Geschlechts. 
Diese „alte“ Definition wurde in den Gleichbehandlungs-Richtlinien aus dem Jahr 2000 durch eine „neue“ Definition abgelöst. Danach „liegt eine mittelbare Diskriminierung vor, wenn dem Anschein nach neutrale Vorschriften, Kriterien oder Verfahren Personen, die einer Rasse oder ethnischen Gruppe angehören, in besonderer Weise benachteiligen können, es sei denn, die betreffenden Vorschriften, Kriterien oder Verfahren sind durch ein rechtmäßiges Ziel sachlich gerechtfertigt, und die Mittel sind zur Erreichung dieses Ziels angemessen und erforderlich. “8

Die RL 2006/54/EG formuliert die Definition an deren Beginn wieder etwas anders. Danach ist mittelbare Diskriminierung „eine Situation, in der dem Anschein nach neutrale Vorschriften, Kriterien oder Verfahren Personen des einen Geschlechts in besonderer Weise gegenüber Personen des anderen Geschlechts benachteiligen können, es sei denn ...".9 Wesentlich ist also, dass die Verwendung eines neutralen Kriteriums Personen bzw. Sachverhalte, die das unzulässige Merkmal aufweisen, in besonderer Weise benachteiligen können.

Der EuGH hat die anderen oben genannten Diskriminierungsverbote aber schon vor Jahrzehnten auch auf mittelbare Diskriminierung erstreckt. In der Literatur wird der Begriff der mittelbaren Diskriminierung denn auch zu Art. 18 AEUV und zu den vier Freizügigkeiten verwendet, insbesondere zur Arbeitnehmerfreizügigkeit. Auch hier bejaht der EuGH seit langem eine mittelbare Diskriminierung dann, wenn sich die nationalen Vorschriften „besonders auf“ Personen mit dem unzulässigen Differenzierungsmerkmal auswirken können. So ist es etwa bei Vorschriften, die sich auf Wanderarbeitnehmer auswirken. ${ }^{10}$ Die Entwürfe zu den Geichbehandlungs-Richtlinien 2000 berufen sich auf dieses Vorbild. ${ }^{11}$

Die praktische Bedeutung eines Verbotes mittelbarer Diskriminierung richtet sich allerdings wesentlich danach, ob die dadurch geschützten Interessen weitergehend auch durch ein Verbot von Beschränkungen geschützt werden. Dies ist nach h.M. bei den vier Freizügigkeiten der Fall. ${ }^{12}$ Bei den Gleichbehandlungs-Richtlinien gibt es hingegen kein „ergänzendes“ Beschränkungsverbot, und auch nicht bei Art. 18

8 Art. 2 Abs. 2 lit. b) Richtlinie 2000/43/EG. Gleichsinnig Art. 2 Abs. 2 lit. b) Richtlinie 2000/78/EG; Art. 2 Abs. 2 Richtlinie 2002/73/EG zur Änderung der Richtlinie 76/207/EWG zur Verwirklichung des Grundsatzes der Gleichbehandlung von Männern und Frauen hinsichtlich des Zugangs zur Beschäftigung, zur Berufsbildung und zum beruflichen Aufstieg sowie in Bezug auf die Arbeitsbedingungen; sowie Art. 2 lit. b) Richtlinie 2004/113/EG.

9 Art. 2 Abs. 1 lit. b) Richtlinie 2006/54/EG.

10 EuGH, Rs. C-237/94, O'Flynn, Slg. 1996, I-02617 Rn. 18-21 m.w.N.; sowie z.B. EuGH, Rs. C-212/05, Hartmann, Slg. 2007, I-6303 Rn. 30. Vgl. jüngst auch EuGH, verb.Rs. C-570/07 und C-571/07, Blanco Pérez und Chao Gómez, Slg. 2010, I-00000 Rn. 119 zur Niederlassungsfreiheit.

11 Insbes. wird EuGH, Rs. C-237/94, O Flynn, Slg. 1996, I-2617 genannt; vgl. Vorschlag für eine Richtlinie zur Festlegung eines allgemeinen Rahmens für die Verwirklichung der Gleichbehandlung in Beschäftigung und Beruf, KOM [1999] 565 endg, ABl. C 177E vom 27.6.2000, S. 42-46.

12 Vgl. nur D. Ehlers, Arbeitnehmerfreizügigkeit, in: ders. (Hrsg.), Europäische Grundrechte und Grundfreiheiten, 3. Aufl., Berlin 2009, $\mathbb{S} 9$ Rn. $81 \mathrm{ff}$. 
AEUV. ${ }^{13}$ Bei den vier Freiheiten hat daher die genaue Umschreibung des Tatbestandes i.w.S. (einschließlich der Frage einer Rechtfertigung) mittelbarer Diskriminierung jedenfalls in der Praxis nicht dieselbe Bedeutung wie bei den anderen Verboten, weil man stets auf das Beschränkungsverbot „ausweichen“ kann. Der EuGH tut dies auch. ${ }^{14}$ In der Literatur wird denn auch festgestellt, dass in der Judikatur des EuGH der Diskriminierungsbegriff reichlich unklar ist und dass sich die Verbote mittelbarer Diskriminierung und von Beschränkungen überschneiden. ${ }^{15}$ Manche vertreten, dass die vier Freizügigkeiten - jedenfalls im Kontext des heutigen Unionsrechtes - nur als Diskriminierungsverbote zu verstehen seien, also nur eingreifen, wenn die grenzüberschreitenden Sachverhalte ungünstiger als die rein nationalen Sachverhalte behandelt werden. ${ }^{16}$ Folgte der EuGH dem, dann müsste die Kategorie der mittelbaren Diskriminierung hier weit stärker und fallgruppenbezogen konkretisiert werden, was in der Folge auch hier die Frage nach der Bedeutung von Kausalität verstärkt stellte.

Im Folgenden wird die Frage des Verhältnisses von mittelbarer Diskriminierung und Kausalität daher primär anhand der Gleichbehandlungs-Richtlinien erörtert, und die Lage bei den vier Freizügigkeiten und Art. 18 AEUV nur ergänzend herangezogen.

\section{Meinungsstand zum Kausalitätserfordernis}

Nach verbreiteter Auffassung setzen sowohl die unmittelbare wie die mittelbare Diskriminierungen einen (objektiven) Kausalzusammenhang zwischen Benachteiligung und unzulässigem Unterscheidungsmerkmal voraus. ${ }^{17}$

Unmittelbare Diskriminierung liegt unbestreitbar nur vor, wenn das unzulässige Merkmal kausal bzw. Ursache für die Schlechterstellung ist, etwa wenn Arbeitskräfte aufgrund der anderen Staatsangehörigkeit schlechter gestellt werden oder das Gesetz

13 Vgl. dazu T. Kingreen, Verbot der Diskriminierung wegen der Staatsangehörigkeit, in: D. Ehlers (Hrsg.), Europäische Grundrechte (Fn. 12), $\$ 13$ Rn. 17 ff.; andernfalls würde Art. 18 AEUV wohl zu einem Grundrecht auf „allgemeine Handlungsfreiheit“ tendieren. Dasselbe gilt in Bezug auf Art. 110 AEUV.

14 Der EuGH wechselt zwischen den Ansätzen der Diskriminierung und des Marktzuganges, und zwar ohne erkennbare Linie; vgl. z.B. die Judikaturanalyse bei T. Kingreen, Die Struktur der Grundfreiheiten des Europäischen Gemeinschaftsrechts, Berlin 1999, S. 40 ff.

15 Vgl. nur T. Kingreen, Grundfreiheiten, in: A. von Bogdandy/J. Bast (Hrsg.), Europäisches Verfassungsrecht, 2. Aufl., Heidelberg 2009, S. 705 (727 ff.) m.w.N.; D. Ehlers, Allgemeine Lehren, in: ders. (Hrsg.), Europäische Grundrechte (Fn. 12), $\$ 7$ Rn. 89.

16 So z.B. Kingreen, Grundfreiheiten (Fn. 15), S. 705 (727 ff.). Tendenziell auch GA Poiares Maduro in seinem SA zu EuGH, verb.Rs. C-158/04 und C-159/04, Alfa Vita Vassilopoulos („Bake-off“), Slg. 2006, I-8153 Rn. 41 ff., auch wenn er u.U. doch über ein bloßes Diskriminierungsverbot hinausgeht.

17 Z.B. Kingreen, Struktur der Grundfreiheiten (Fn. 14), S. 84 f.; S. Plötscher, Der Begriff der mittelbaren Diskriminierung im Europäischen Gemeinschaftsrecht, Berlin 2003, S. 51 ff.; M. Schlachter, Der Begriff der Diskriminierung im Europäischen Gemeinschaftsrecht, RdA 2004, S. 190 (190); dies., Probleme der mittelbaren Benachteiligung im Anwendungsbereich des Artikel 119 EGV, NZA 1995, S. 393 (396); H. Oetker, in: R. Richardi/H. Wissmann/O. Wlotzke/H. Oetker (Hrsg.), Münchener Handbuch zum Arbeitsrecht, 3. Aufl., München 2009, $\$ 14$ Rn. 50; C. Tobler, Indirect Discrimination, Antwerpen-Oxford: Intersentia 2005, S. 354 ff.; M. Foster, Causation in Context, Michigan Journal of International Law 2002, S. 265 (319). 
Arbeitnehmer über oder unter einem bestimmten Lebensalter schlechter stellt. $\mathrm{Zu}$ den Gleichbehandlungs-Richtlinien sagen manche, dieses Kausalitätserfordernis folge dort aus den Worten „aufgrund“ oder „wegen“, das die RL bei der Definition verwenden. ${ }^{18}$ Gleiches wird wie gesagt auch zu Art. 3 Abs. 3 des deutschen GG vertreten. ${ }^{19}$

Bei der mittelbaren Diskriminierung ist der Kausalzusammenhang zwischen unzulässigem Merkmal und Schlechterstellung hingegen nicht wirklich klar, weil sie definitionsgemäß ja nicht daran, sondern an ein neutrales Unterscheidungsmerkmal anknüpft. Im Schrifttum zur mittelbaren Diskriminierung wird der Kausalzusammenhang zwischen unzulässigem Differenzierungskriterium und Benachteiligung meist nur behauptet und verlangt, aber kaum näher erörtert. ${ }^{20}$ Auch die mitunter anzutreffende Aussage, die (festgestellte) mittelbare Benachteiligung von Personen mit Ausprägung des unzulässigen Unterscheidungsmerkmals begründe bloß eine (durch sachliche Rechtfertigung widerlegbare) Kausalitätsvermutung, ${ }^{21}$ sagt wenig über die Beschaffenheit des (wenn auch nur vermuteten) Kausalzusammenhanges aus.

Ausführlicher diskutiert das Schrifttum zur mittelbaren Diskriminierung meist nur die gedanklich nachgelagerte Frage, ob die Rechtfertigungsmöglichkeit einer mittelbaren Benachteiligung ein Element der (welcher?) Kausalitätsprüfung sei. ${ }^{22}$ Nach der einen Auffassung seien bei Rechtfertigung einer mittelbaren Benachteiligung schon der Kausalzusammenhang zwischen unzulässigem Differenzierungskriterium und Benachteiligung und damit auch der Tatbestand der Diskriminierung im Sinne einer Benachteiligung wegen des unzulässigen Differenzierungsmerkmals zu verneinen. ${ }^{23}$ Andere sehen das Rechtsfertigungselement hingegen als einen echten Rechtfertigungsgrund, dessen Vorliegen zwar nicht den Kausalzusammenhang zwischen un-

18 So liegt eine unmittelbare Diskriminierung gem Art. 2 Abs. 2 lit a) RL 2000/78/EG vor, „wenn eine Person wegen eines der in Artikel 1 genannten Gründe in einer vergleichbaren Situation eine weniger günstige Behandlung erfährt, ....“. Die RL 2000/43/EG und die späteren RL verwenden anstelle von „wegen“ die Präposition „aufgrund“. In beiden Präpositionen kann man das Erfordernis der Kausalität ausgedrückt sehen.

19 Vgl. die Nachweise bei Fn. 1.

20 Der EuGH spricht in seinen Entscheidungen zur mittelbaren Diskriminierung im Rahmen der Gleichbehandlung die Kausalitätsfrage meist - wenn überhaupt - so nur indirekt an, und zwar als Einschränkung der sachlichen Rechtfertigungsmöglichkeit: Danach ist z.B. eine mittelbare Benachteiligung nur zulässig, wenn sie objektiv gerechtfertigt ist, und nichts mit einer Diskriminierung auf Grund des Geschlechts (oder eines anderen unzulässigen Unterscheidungsmerkmals) zu tun hat (stereotyp wiederholter Rechtssatz in der st. Rsp.).

21 So z.B. Schlachter, Mittelbare Benachteiligung (Fn. 17), S. 396; dies., in: R. Müller-Glöge/U. Preis (Hrsg.), Erfurter Kommentar zum Arbeitsrecht' 10. Aufl., München 2010, \$ 3 AGG, Rn. 6; dies., in Erfurter Kommentar (Fn. 21), Art. 141 EGV, Rn. 15 („,erhöhte Wahrscheinlichkeit“); K. Adomeit/J. Mohr, Kommenter zum Allgemeinen Gleichbehandlungsgesetz, Stuttgart 2007, S. 272.

22 Vgl. eingehend Tobler, Indirect Discrimination (Fn. 17), S. 254 ff.; D. Schiek/L. Waddington/M. Bell, Cases, Materials and Texts on National, Supranational and International Non-Discrimination Law, Oxford: Hart Publishing 2007, 3.5.2.A.

23 Nachweise bei Tobler, Indirect Discrimination (Fn. 17), S. 255. 
zulässigem Unterscheidungsmerkmal und Benachteiligung (und damit auch nicht den Vorwurf der Diskriminierung oder Benachteiligung wegen dieses Merkmals) beseitigt, sondern die Benachteiligung (einschließlich ihrer kausalen Verknüpfung mit dem an sich unzulässigem Differenzierungsgrund) ausnahmsweise wegen sachlicher Rechtfertigung und Erforderlichkeit erlaubt. ${ }^{24}$

Abgesehen davon, dass die praktische Bedeutung dieser Diskussion von vielen (wohl zu Recht) als gering eingeschätzt wird, ${ }^{25}$ ist diese Diskussion noch einem anderen Kritikpunkt ausgesetzt. Sie verdeckt und überspielt die gedanklich vorgelagerte und für Struktur und Funktion des Verbots mittelbarer Diskriminierungen wichtigere Frage, ob und gegebenenfalls in welcher Form das Tatbestandselement der mittelbaren Benachteiligung und damit auch der Tatbestand der mittelbaren Diskriminierung überhaupt einen Kausalzusammenhang zwischen unzulässigem Differenzierungsmerkmal und Benachteiligung verlangt. Erst wenn dies geklärt ist, kann sinnvoll danach gefragt werden, ob und inwieweit sich die Rechtfertigung auf diesen (einen) Kausalzusammenhang auswirkt.

\section{B. Besteht bei mittelbarer Diskriminierung ein Kausalzusammenhang zwischen unzulässigem Merkmal und Schlechterstellung?}

\section{Mittelbare Diskriminierung aus dem Blickwinkel unterschiedlicher Kausalitätskonzepte}

Diskriminierungsverbote wenden sich anders als ein allgemeiner Gleichheitssatz nicht schon gegen jede unsachliche Differenzierung, sondern nur gegen Ungleichbehandlungen durch Anknüpfen an bestimmte Merkmale. Sie scheinen auf den ersten Blick daher schon aufgrund ihrer Struktur einen Kausalzusammenhang zwischen Benachteiligung und unzulässigem Unterscheidungsmerkmal vorauszusetzen. Die Kausalität wird üblicherweise nach dem conditio-Test (but-for-test) oder nach der

24 So insbes. Tobler, Indirect Discrimination (Fn. 17), S. 258. Die Frage, ob und wie sich die Rechtfertigung einer mittelbaren Benachteiligung auf den Kausalzusammenhang zwischen Benachteiligung und unzulässigem Unterscheidungsmerkmal auswirkt, deckt sich im Übrigen nicht unbedingt (vollständig) mit der Frage, ob die Rechtfertigungsmöglichkeit eine Tatbestandseinschränkung oder ein Rechtfertigungsgrund ist. Denn auch die Einordnung als Tatbestandseinschränkung (wofür etwa der Wortlaut der Definitionen der mittelbaren Diskriminierung in den Gleichbehandlungs-Richtlinien spricht; vgl. z.B. Art. 2 Abs. 1 lit. b) RL 2006/54/EG; Art. 2 Abs. 2 lit. b) RL 2000/43/EG; Art. 2 Abs. 2 RL 97/80/ EG) sagt nur, dass im Fall einer rechtfertigbaren mittelbaren Benachteiligung nicht von mittelbarer Diskriminierung gesprochen werden kann, nicht aber, dass kein Kausalzusammenhang zwischen Benachteiligung und unzulässigem Unterscheidungsmerkmal besteht.

25 N. Bamforth, Setting the Limits of Anti-Discrimination Law, in: J. Dine/B. Watt (Hrsg.), Discrimination Law. Concepts. Limitations, and Justifications, London/New York: Longman 1996, S. 49 (50); O. Steiner, Das Verbot der Diskriminierung aufgrund des Geschlechts im Erwerbsleben, München 1999, S. 273; in diese Richtung letztlich auch Tobler, Indirect Discrimination (Fn. 17), S. 254, nach der aber immerhin einige Unterschiede bestehen; vgl. dazu unten Fn. 56. 
Theorie der gesetzmäßigen Bedingung geprüft. ${ }^{26}$ Der conditio-Test fragt, ob das als Ursache in Betracht Kommende conditio sine qua non für das als Folge in Betracht Kommende (hier: Schlechterstellung) ist. Die Theorie der gesetzmäßigen Bedingung fragt, ob auf das als Ursache in Betracht Kommende mit einem zeitlich nachfolgenden Ereignis „gesetzmäßig“, also auf Grund einer allgemeinen Kausalhypothese, verbunden ist. Entgegen der Ansicht mancher besteht zwischen conditio-Test und Theorie der gesetzmäßigen Bedingung kein Gegensatz, vielmehr handelt es sich um zwei Aspekte derselben Überlegung, von denen jeder den anderen implizit enthält. ${ }^{27}$

Wie erwähnt, verlangt nur der Tatbestand der unmittelbaren Diskriminierung ausdrücklich den fraglichen Kausalzusammenhang. Der Tatbestand der mittelbaren Diskriminierung stellt hingegen primär darauf $a b$, dass ein - vielleicht nur scheinbar - neutrales Unterscheidungsmerkmal verwendet wird. Zwischen diesem neutralen Kriterium und der Schlechterstellung besteht nun ohne Zweifel ein Kausalzusammenhang. Allerdings ist hier nicht dieser Kausalzusammenhang von Interesse, sondern jener zwischen unzulässigem Unterscheidungsmerkmal und Schlechterstellung. Dieser Kausalzusammenhang wird durch den zuerst genannten nun nicht etwa ausgeschlossen, weil ein Ereignis ja stets durch eine Vielzahl von Umständen verursacht worden sein kann und meist auch ist.

Bei den hier interessierenden Konstellationen, der Schlechterstellung durch ein dem Anschein nach neutrales Merkmal, scheint das unzulässige Merkmal aber nun gerade nicht Ursache der Schlechterstellung im Einzelfall zu sein. Stellt etwa der Arbeitgeber nur Arbeitnehmer ohne Kinderbetreuungspflichten ein, so liegt darin nach h.A. eine mittelbare Benachteiligung von Frauen, wenn und weil diese typischerweise die Kinderbetreuung übernehmen oder zumindest organisieren. Nach den traditionellen Kausalitätskriterien scheint man aber nicht sagen zu können, dass hier das Geschlecht Ursache für die Benachteiligung ist, wenn und weil auch Männer mit Kinderbetreuungspflichten nicht zum Zug kommen. Dies gilt auch dann, wenn die mittelbare Benachteiligung der weiblichen Bewerberinnen nicht rechtfertigbar und daher eine (unzulässige) Geschlechterdiskriminierung ist.

26 Diese beiden Kausalitätstheorien sind die in den Mitgliedstaaten vorherrschenden; vgl. J. Spier/O.A. Haazen, Comparative Conclusions, in: Spier (Hrsg.) Unification of Tort Law: Causation, The Hague: Kluwer Law Internatinal 2000, S. 126 (126 ff.); H.L.A. Hart/ T. Honoré, Causation in the Law, Oxford: Oxford University Press, S. 442 ff.; vgl. zu beiden Theorien näher z.B. auch R. Rebhahn, Staatshaftung wegen mangelhafter Gefahrenabwehr, Wien: Manz 1997, S. 545 ff. Das Gemeinschaftsrecht kennt keine autonome Regel zur Kausalitätsprüfung, der EuGH bedient sich wohl überwiegend des but-for-Tests; vgl. dazu zuletzt die Judikaturanalyse von I.C. Durant, Causation, in: H. Koziol/R. Schulze (Hrsg.), Tort Law of the European Community, Wien: Springer 2008, S. 47 (47 ff.).

27 Auch der conditio-Test kommt nicht ohne das Konzept der gesetzmäßigen Bedingung aus, weil die Beantwortung der Frage, ob B bei Wegdenken von A entfällt, Erfahrungswissen über gesetzmäßige Zusammenhänge zwischen A und B voraussetzt. Vgl. zum Ganzen Rebhahn, Staatshaftung (Fn. 26), S. $545 \mathrm{ff}$. 
Das eben Gesagte gilt unabhängig davon, ob man die Kausalitätsprüfung nach den conditio-Test vornimmt oder nach der Theorie der gesetzmäßigen Bedingung. Es ist offenkundig, dass im obigen Beispiel die Schlechterstellung von Bewerbern mit Kinderbetreuungspflichten auch bei Wegdenken des (weiblichen) Geschlechts nicht entfällt. Damit scheitert aber zugleich die Annahme eines Kausalzusammenhanges nach dem Konzept der gesetzmäßigen Bedingung. Nach diesem Konzept scheint für die Annahme eines entsprechenden Kausalzusammenhanges zwar zunächst die hier (als statistisch fundiert oder plausibel vermutet) vorausgesetzte Annahme zu sprechen, dass beim Abstellen auf Kinderbetreuungspflichten der Nachteil B (Nichteinstellung) weitaus häufiger mit $\mathrm{A}$ (weibliches Geschlecht) verknüpft ist als mit $\mathrm{C}$ (männliches Geschlecht). Daraus ließe sich aber nur dann eine sinnvolle Kausalhypothese zwischen A und B ableiten, wenn zugleich gesagt werden könnte, dass der Nacheil B ohne A bei sonst gleichen Umständen (hier also Kinderbetreuungspflichten auch bei C) nicht oder doch weniger häufig folgt. Es lässt sich auch nicht sagen, dass A notwendig zu B führt, weil nicht alle Frauen Kinderbetreuungspflichten haben.

Ähnliches gilt auch für die (heute allerdings kaum noch gebräuchliche) wertende Kausalitätsprüfung nach dem Konzept der wesentlichen Bedingung. ${ }^{28}$ Danach ist zwar im Einzelfall auf eine ganze Fülle von Umständen oder Ereignissen zu blicken, und daraus nach einer wertenden Betrachtung die wesentliche Ursache für ein Ereignis auszuwählen, sodass das Erfordernis der conditio sine qua non auf den ersten Blick in den Hintergrund zu treten scheint. Eine solche Betrachtung könnte dazu verleiten, bei mittelbaren Diskriminierungen das unzulässige Unterscheidungsmerkmal zumindest wertungsmäßig als eine Ursache für die Benachteiligung anzusehen und bei fehlender Rechtfertigung sogar als die wesentliche Ursache. Dies wäre allerdings insofern ein Trugschluss, als letztlich auch das Konzept der wesentlichen Bedingung nicht ohne conditio-test auskommt, wenn daraus eine Kausalzusammenhang im Sinne eines echten Ursachenzusammenhanges abgeleitet werden soll. Obwohl dies selten ausdrücklich gesagt wird, ist auch nach dem Konzept der wesentlichen Bedingung nämlich zunächst anhand des conditio-Tests zu bestimmen, welche Ereignisse und Umstände für ein bestimmtes Ereignis ursächlich sind. Erst dann kann daraus anhand einer wertenden Betrachtung die wesentliche Ursache bestimmt werden. Die Theorie der wesentlichen Bedingung kann daher niemals zu einer weiteren Zurechnung als der conditio-Test führen, sondern immer nur zu einer engeren; auch

28 In den Mitgliedstaaten werden durchaus auch noch Kausalitätskonzepte mit wertenden Betrachtungen und Beschränkungen verwendet. Zu nennen ist etwa die „Theorie der wesentlichen Bedingung“ im Recht der Gesetzlichen Unfallsversicherung; dazu z.B. E. A. Kramer, Das Kausalitätsproblem im österreichischen und schweizerischen Unfallversicherungsrecht, in: O. Martinek (Hrsg.), Arbeitsrecht und soziale Grundrechte. Festschrift Hans Floretta zum 60. Geburtstag, Wien: Manz 1983, S. 683 (683 ff.). Vgl. allgemein Rebhahn, Staatshaftung (Fn. 26), S. 546 ff.; Hart/Honoré, Causation ${ }^{(\text {Fn. 26) }}{ }^{\text {, }}$ S. $431 \mathrm{ff}$. Im Deliktsrecht waren früher, als andere Schranken der Zurechnung noch weniger entwickelt waren, Einschränkungen der Haftung unter dem Titel fehlender Kausalität häufiger und wichtiger als heute. 
sie begründet daher im obigen Beispiel der Nichteinstellung wegen Kinderbetreuungspflichten keinen Kausalzusammenhang zwischen Geschlecht und Nichteinstellung.

Das eben Gesagte lässt sich auch für andere Fälle zeigen, in denen eine mittelbare Benachteiligung angenommen wird. So wird eine Benachteiligung von Teilzeitarbeitskräften als mittelbare Benachteiligung aufgrund des Geschlechts angesehen, obwohl weder nach den generalisierenden Kausalitätskonzepten (conditio und gesetzmäßige Bedingung) noch nach dem individualisierenden Konzept der wesentlichen Bedingung ein Kausalzusammenhang zwischen Benachteiligung und dem unzulässigem Unterscheidungsmerkmal Geschlecht besteht. Dasselbe gilt für den Fall, dass eine Bewerbung wegen schlechter Kenntnisse der Landessprache abgelehnt wird; auch hier ist die landesfremde ethnische Herkunft nicht Ursache im Sinne der üblichen Kausalitätsformeln.

Auch wenn in den drei genannten Fällen somit kein Kausalzusammenhang zwischen Benachteiligung und unzulässigem Kriterium besteht, so besteht doch eine - hohe Korrelation zwischen diesen beiden Elementen: Einheimische sprechen die Landessprache üblicherweise (wenn auch weder notwendig noch stets) besser als Personen fremder ethnischer Herkunft; Frauen betreuen Kinder weitaus häufiger als Männer und arbeiten weitaus häufiger Teilzeit als Männer. Die hohe Korrelation zwischen zwei „Variablen“ (z.B. Frau und Teilzeit) begründet aber weder allgemein noch hier konkret einen Kausalzusammenhang.

Der Gesetzgeber der Gleichbehandlungs-Richtlinien scheint diese „Entfernung“ der mittelbaren Diskriminierung von der Kausalität nun bemerkt zu haben. Die beiden Gleichbehandlungs-Richtlinien aus 2000 enthalten noch eine übergreifende Bestimmung unmittelbar vor den Definitionen der beiden Diskriminierungsformen. Art. 2 Abs. 1 Richtlinie 2000/78/EG sagt dort: „Im Sinne dieser Richtlinie bedeutet, Gleichbehandlungsgrundsatz', dass es keine unmittelbare oder mittelbare Diskriminierung wegen eines der in Artikel 1 genannten Gründe geben darf. " 29 Die andere RL aus 2000 (RL 2000/43/EG) sagt hier „aus Gründen“ anstelle von „wegen“. Sieht man, wie oben dargelegt, im Normtext zur unmittelbaren Diskriminierung in „wegen“ und „aufgrund“ die Anordnung des Kausalitätserfordernisses, dann müsste dieses danach auch für die mittelbare Diskriminierung bestehen. In den beiden späteren RL zur Gleichbehandlung aufgrund des Geschlechtes (RL 2004/113/EG; RL 2006/54/EG) fehlt nun die übergreifende Definition. Dies ist durchaus konsequent.

Die verbreitete Auffassung, wonach auch mittelbare Diskriminierungen einen Kausalzusammenhang zwischen Benachteiligung und unzulässigem Unterscheidungs- 
merkmal voraussetzen, findet jedenfalls für den Bereich der GleichbehandlungsRichtlinien durch die traditionellen Kausalitätskonzepte keine Bestätigung.

Dasselbe gilt auch bei den Freizügigkeiten. Der EuGH hat eine mittelbare Diskriminierung aufgrund der Staatsangehörigkeit etwa im Urteil Angonese bejaht. ${ }^{30}$ Ein Arbeitgeber verlangte von Bewerbern den Nachweis von Sprachkenntnissen, und dieser Nachweis konnte ausschließlich durch ein einziges Diplom erfolgen, das nur in einer einzigen Provinz Italiens ausgestellt werden konnte. Da diese Einschränkung auch die Bewohner aller anderen - über 50 - Provinzen Italiens traf, war die fremde Staatsangehörigkeit offenkundig keine conditio für den Nachteil noch begründete sie eine gesetzmäßige Bedingung.

Auch eine Kausalitätsprüfung anhand den von der deutschen Doktrin zu den besonderen Diskriminierungsverboten des Art. 3 GG entwickelten „Kausalitätskonzepten“ ändert nichts am bisher Gesagten. ${ }^{31}$ Dies gilt sowohl für die Deutung der Diskriminierungsverbote als Anknüpfungsverbote, wonach grundsätzlich jede tatbestandliche Anknüpfung an ein verpöntes Merkmal als Unterscheidungskriterium untersagt ist, als auch für das heute kaum noch vertretene Ausschließlichkeits- bzw. Finalitätsmodell, wonach bloß Differenzierungen „allein “ oder „gerade wegen“ des verpönten Merkmals unzulässig sind. Nach beiden Deutungen setzt das Diskriminierungsverbot einen Kausalzusammenhang im Sinne der oben dargelegten traditionellen Kausalitätstheorien voraus und erfasst daher ebenfalls nur unmittelbare Diskriminierungen, nicht aber mittelbare. Auch die häufig vetretene Deutung der Diskriminierungsverbote als Begründungsverbote, wonach die Ungleichbehandlung ohne Rückgriff auf das verpönte Merkmal begründbar sein muss, hilft für die hier interessierende Fragestellung nicht weiter. Lässt man bereits die formale Anknüpfung an das neutrale Kriterium als ausreichende Begründung für die (faktische) Schlechterstellung von Personen mit verpöntem Merkmal ausreichen, so sind mittelbare Diskriminierungen weiterhin nicht vom Diskriminierungsverbot erfasst. Verlangt man hingegen für die faktische Schlechterstellung eine echte, materielle Rechtfertigung, so erstreckt sich das Verbot zwar auch auf mittelbare Diskriminierungen, über den hier interessierenden Kausalzusammenhang zwischen verpöntem Merkmal und Schlechterstellung ist damit aber wieder nichts ausgesagt.

Man sollte daher zumindest die Deutung als Begründungsverbot besser gar nicht als besonderes „Kausalitätskonzept“ bezeichnen, wenn und weil Kausalität nach üblichem Sprachgebrauch einen objektiven Ursachenzusammenhang im Sinne des conditio-Tests bzw. der Theorie der gesetzmäßigen Bedingung voraussetzt. Hingegen bewirkt das Finalitäts- bzw. Ausschließlichkeitsmodell bloß eine Einschränkung der

30 EuGH, Rs. C-281/98, Angonese, Slg. 2000, I-4139.

31 Anknüpfungsverbot, Ausschließlichkeits- bzw. Finalitätsmodell, Begründungsverbot; vgl. zu diesen Konzepten vor allem Heun (Fn. 1), Art. 3 Rn. 119 ff.; Dürig/Scholz (Fn. 1), Art 3 Rn. 133 ff. 
durch objektiven Kausalzusammenhang begründeten Zurechnung durch subjektive und wertende Momente. Sofern im Folgenden bloß von Kausalität gesprochen wird, ist damit jedenfalls lediglich ein objektiver Ursachenzusammenhang nach dem conditio-Test bzw. nach der Theorie der gesetzmäßigen Bedingung gemeint.

\section{Sonderfälle mittelbarer Diskriminierung}

Zu überlegen bleibt, ob in manchen Fällen, insbesondere zu den Freizügigkeiten, der fragliche Kausalzusammenhang nicht doch bejaht werden könnte, wenn man die Fragestellung etwas „umformuliert“ und nur fragt, ob das unzulässige Merkmal auch wenn es nicht conditio ist - den Nachteil notwendig nach sich zieht.

Man denke etwa an das nationale Verbot des Verkaufes von Medikamenten über das Internet, das der EuGH im DocMorris-Urteil behandelt hat. ${ }^{32}$ Der Standort des Verkäufers in einem anderen Mitgliedstaat ist hier nicht Ursache im Sinne des conditio-Tests, weil das Verbot jede vom Käufer räumlich entfernte Apotheke trifft, auch im selben Mitgliedstaat. Allerdings bringt der ausländische Standort das Eingreifen des Verbotes notwendig mit sich; ausländische Apotheken können nur im Wege des Versandhandels verkaufen. Man könnte fragen, ob der ausländische Standort (wenn dieser schon nicht conditio ist) deshalb zumindest „gesetzmäßige Bedingung “ für die Rechtsfolge des Verkaufsverbotes ist. Der ausländische Standort einer Apotheke ist nun ohne Zweifel Grund dafür, dass diese im Regelungsstaat Medikamente nicht verkaufen dürfen, und es besteht auch eine hohe Korrelation mit dem Verkaufsverbot. Beides macht den ausländischen Standort, jedenfalls wenn man den traditionellen Vorstellungen folgt, aber noch nicht zur Ursache für das Verkaufsverbot i.S.d, Konzeptes der gesetzmäßigen Bedingung, weil diese Rechtsfolge nach der gegebenen Norm eben auch bei reinen Inlandsfällen eintritt. Auch eine gesetzmäßige Bedingung kann danach letztlich nur bejaht werden, wenn die Rechtsfolge entfällt falls man sich das unzulässige Merkmal wegdenkt. ${ }^{33}$ Dies wäre aber nur bei einem Internet-Verkaufsverbot spezifisch für ausländische Apotheken der Fall. ${ }^{34}$ Im Rahmen der mittelbaren Diskriminierung führt die „Umformulierung“ also zu keinem anderen Ergebnis.

Ebenso ist es in anderen Fällen. Jüngst hat der EuGH eine mittelbare Diskriminierung zur Niederlassungsfreiheit im Urteil Blanco Pérez bejaht. ${ }^{35}$ Eine Vorschrift begüns-

32 Vgl. dazu EuGH, Rs. C-322/01, Dt. Apotherkerverband, Slg. 2003, I-14887.

33 Der EuGH hat, insbes. in Rn. 74 des Urteils, denn auch keinen Kausalzusammenhang behauptet, sondern spricht von „, besonderer Beeinträchtigung“. Gleich darauf betont er, dass damit der Marktzugang für ausländische Erzeugnisse stärker behindert wird als für inländische. Dies zeigt, wie wenig deutlich der EuGH hier zwischen mittelbarer Diskriminierung und Beschränkungsverbot trennt.

34 Dies wird verdeckt, wenn man das Internet-Verkaufsverbot schon (und nur) in Bezug auf ausländische Apotheken als „Schlechterstellung“ bezeichnet. Bei der gegebenen Norm dürfte man nur von einer Schlechterstellung nicht am Ort des Käufers ansässiger Apotheken sprechen.

35 EuGH, verb.Rs. C-570/07 und C-571/07, Blanco Pérez und Chao Gómez, Slg. 2010, I-00000. 
tigte bei der Auswahl zum Zugang eines Berufes (Apotheker) Vordienstzeiten in einer bestimmten Region eines Mitgliedstaates. Auch hier ist die Staatsangehörigkeit zu einem anderen Mitgliedstaat nicht conditio für die Schlechterstellung, weil Vordienstzeiten in anderen Regionen des betreffenden Mitgliedstaates ebenfalls schlechter gestellt waren. Allerdings waren Vordienstzeiten im Ausland notwendig schlechter gestellt. Gleichwohl ist nach dem Gesagten die ausländische Vortätigkeit keine gesetzmäßige Bedingung für die Schlechterstellung. Erlaubt das nationale Recht das Aufbacken aller tief gefrorenen Backwaren („Bake-Off“) nur jenen, die eine Gewerbebefugnis für das Bäckereigewerbe haben, so ist eine ausländische Herkunft der Backwaren offenkundig nicht conditio für das Verbot, Backwaren ohne Gewerbebefugnis fertig zu backen. ${ }^{36}$ Auch wenn das Verbot ausländische tief gefrorene Backwaren notwendig trifft, ist die ausländische Herkunft des Produktes keine "gesetzmäßige Bedingung“ i.e.S. für das Verbot, schon weil das Verbot auch unbegrenzt viele im Inland hergestellte Bake-off Produkte trifft.

Die juristischen Konzepte der Kausalität sind - anders als jenes der naturwissenschaftlichen Kausalität - allerdings keine präpositiv feststehenden Größen, sondern rechtliche Regeln, die der Ausgestaltung durch das Recht zugänglich sind. Auch im Deliktsrecht wird das Kausalitätserfordernis richtiger Auffassung nach durch den Normzweck beeinflusst. ${ }^{37}$ Es wäre daher denkbar, dass der Unionsgesetzgeber zu seinen Diskriminierungsverboten anordnete: Ein unzulässiges Merkmal ist kausal für eine (Rechts-)Folge, wenn es diese notwendig nach sich zieht, auch wenn die geprüfte Regelung sie an ein anderes Merkmal knüpft. Sinnvoller als eine terminologische Anordnung wäre es allerdings, wenn das Unionsrecht die eben genannte Konstellation bei der mittelbaren Diskriminierung - insbesondere bei den Regeln für transnationale Sachverhalte - gesondert bedenkt (was derzeit wohl kaum der Fall ist).

\section{Vermutung einer - kausalen - unmittelbaren Diskriminierung?}

Vor diesem Hintergrund stellt sich dann die Frage, wie sich die verbreitete Auffassung erklären lässt, wonach mittelbare Diskriminierungen einen Kausalzusammenhang zwischen unzulässigem Unterscheidungsmerkmal und Benachteiligung voraussetzen. Diese Auffassung lässt sich wohl am besten historisch erklären, und zwar mit der ursprünglichen Funktion des Verbots mittelbarer Diskriminierungen im Gemeinschaftsrecht als Hilfsmittel zur Aufdeckung unmittelbarer Diskriminierungen.

Die besonderen Diskriminierungsverbote wurden zuerst als bloßes Verbot einer Unterscheidung direkt nach dem unzulässigen Differenzierungsmerkmal verstanden. Verboten waren bloß Maßnahmen, die ausdrücklich oder zumindest offenkundig

36 Vgl. EuGH, verb.Rs. C-158/04 und C-159/04, Alfa Vita Vassilopoulos („Bake-off“), Slg. 2006, I-8153. Der EuGH hat eine Verletzung der Warenverkehrsfreiheit bejaht.

37 Vgl. Rebhahn, Staatshaftung (Fn. 26), S. 543 ff. 
nach dem verbotenen Unterscheidungsmerkmal differenzierten („,overt discrimination“); also Maßnahmen, die schon selbst einen entsprechenden Kausalzusammenhang zwischen Benachteilung und unzulässigem Merkmal beinhalten. Unter Berufung auf eine Entscheidung des US Supreme Court aus dem Jahr 1971, ${ }^{38}$ in welcher erstmals auch ein Verhalten mit lediglich diskriminierenden Auswirkungen („disparat impact“ im Gegensatz zu „disparate treatment“) untersagt wurde, hat der EuGH die Diskriminierungsverbote aber schon bald auf mittelbare Diskriminierungen ausgedehnt. Diese erweiternde Auslegung des Diskriminierungsbegriffes im Gemeinschaftsrecht wurde zuerst zu den Grundfreiheiten entwickelt, und zwar ausgehend von den Maßnahmen gleicher Wirkung bei der Warenverkehrsfreiheit. ${ }^{39} 1981$ hat der EuGH die mittelbare Diskriminierung dann erstmals als vom Verbot des Art. 119 alt EG (Entgeltgleichheit) erfasst angesehen, ${ }^{40}$ und diese Judikaturlinie dann kontinuierlich auch auf die anderen Diskriminierungsverbote erstreckt.

Mit dieser Ausweitung des Diskriminierungsbegriffes verfolgte der EuGH (anders als die US-amerikanische Rechtsprechung) zunächst aber eher keine eigenständige materielle Zwecksetzung zur Beseitigung tatsächlich bestehender Nachteile bestimmter Personengruppen. Vielmehr diente das Verbot mittelbarer Diskriminierungen eher als prozessuales Hilfsmittel zum erleichterten Nachweis (verdeckter) unmittelbarer Diskriminierungen. ${ }^{41}$ Anders gesagt beinhaltet nach diesem Ansatz jedes Verbot der unmittelbaren Diskriminierung im Kern auch ein mittelbares Diskriminierungsverbot, wodurch verhindert werden soll, dass durch Vorschieben neutraler Unterscheidungsmerkmale letztlich der gleiche Effekt erzielt wird wie bei unmittelbarer Differenzierung nach dem verbotenem Unterscheidungsmerkmal. Die mittelbare Diskriminierung ist dann nichts anderes als ein Unterfall der Gesetzesumgehung. ${ }^{42}$

Der EuGH sagt das zwar nicht ausdrücklich, die älteren Entscheidungen zur mittelbaren Diskriminierung deuten aber klar in diese Richtung. So spricht der EuGH in einer der ersten Grundsatzentscheidungen zur mittelbaren Diskriminierung (Sotgiu) aus dem Jahr 1974 davon, dass das Diskriminierungsverbot „auch versteckte Formen einer unmittelbaren Diskriminierung verbietet“ (Rn 11), wobei diese Auslegung geboten sei, um die „rechtliche und tatsächliche Gleichbehandlung der Arbeitnehmer ... zu wahren“. ${ }^{43}$ Der EuGH versteht hier das Verbot der mittelbaren Diskriminie-

38 US SC Griffs vs Duke Power Co, 28 L Ed $2^{\text {nd }} 158$.

39 Vgl. zur Entwicklung die eingehende Analyse bei Tobler, Indirect Discrimination (Fn. 17), S. 102.

40 EuGH, Rs. 96/80, Jenkins, Slg. 1981, 911.

41 Vgl. dazu eingehend Adomeit/Mohr, AGG (Fn. 21), S. 274 ff.; Plötscher, Diskriminierungsbegriff (Fn. 17), S. 54; C. Blomeyer, Das Verbot der mittelbaren Diskriminierung gemäss Art. 119 EGV. Seine Funktion im deutschen Arbeitsrecht, Baden-Baden 1994, S. $97 \mathrm{f}$.

42 So etwa Plötscher, Diskriminierungsbegriff (Fn. 17), S. 61; Temming, Altersdiskriminierung (Fn. 1), S. 440.

43 EuGH, Rs. 152/73, Sotgiu, Slg. 1974, 153 Rn. 12. Diese Formel wird in den (älteren) Folgentscheidungen zur mittelbaren Diskriminierung ständig wiederholt; z.B. EuGH, Rs. 61/77, Kommission/Irland, Slg. 1978, 417; Rs. 137/78, Cram/Toia, Slg. 1979, 2645. 
rung im Ergebnis offenbar als Hilfsmittel zur effektiven Durchsetzung unmittelbarer Diskriminierungsverbote, die auch nicht durch die Verwendung anscheinend neutraler Differenzierungskriterien umgangen werden dürfen.

Versteht man nach dem bisher Gesagten die Rechtsfigur der mittelbaren Diskriminierung daher nicht als eigenständiges Rechtsinstitut, sondern als bloße Beweiserleichterung zum Nachweis verschleierter unmittelbarer Diskriminierungen, so muss man dann für die mittelbare Diskriminierung wohl ebenso einen Kausalzusammenhang zwischen unzulässigem Unterscheidungsmerkmal und Benachteilung verlangen wie für die unmittelbare. Dies trifft jedenfalls für die eben dargelegte Konzeption von der mittelbaren Diskriminierung als bloße Beweiserleichterung zu, aber wohl auch für jene eines bloßen Umgehungsverbotes. Diese Konzeptionen bedeutet ja nichts anderes, als dass mit Bejahung einer mittelbaren Diskriminierung zugleich gesagt wird, dass eine unmittelbare Diskriminierung und damit notwendig auch der hier interessierende Kausalzusammenhang „ausreichend“ nachgewiesen wurden. Der Unterschied zur (offenen) unmittelbaren Diskriminierung besteht dann letztlich nur noch in der Beweislastverteilung hinsichtlich des (hier gerade nicht offenkundigen) Kausalzusammenhanges, was aber hinsichtlich des Kausalzusammenhanges als Beweisthema grundsätzlich keinen Unterschied macht.

Wirkt sich also eine dem Anschein nach neutrale Regelung spezifisch zum Nachteil von Personen aus, bei denen das unzulässige Differenzierungsmerkmal vorliegt, so begründet dies die Vermutung eines Kausalzusammenhanges zwischen unzulässigem Merkmal und Benachteiligung. Streng genommen kann zwar nur die Vermutung aufgestellt werden, dass das unzulässige Merkmal kausal für die Verwendung der typischerweise benachteiligenden Maßnahme oder Regelung ist, nicht auch dass sie kausal für die tatsächliche Benachteiligung im konkreten Einzelfall ist (für die Benachteiligung im Einzelfall ist vielmehr wie gezeigt das neutrale Kriterium Ursache im Sinne des conditio-Tests). Dieser Unterschied zum Kausalzusammenhang bei unmittelbarer Diskriminierung ist im gegebenen Zusammenhang aber vernachlässigbar und braucht daher nicht weiter verfolgt zu werden. ${ }^{44}$

Wichtiger ist hingegen, dass nach der dargelegten Konzeption der potentiell Diskriminierende die (im Detail wie auch immer formulierte) Kausalitätsvermutung durch Sachgründe widerlegen kann, indem er dartut, dass eben nicht das unzulässige Unterscheidungsmerkmal bzw. die in diese Richtung spezifisch benachteiligende Wirkung einer Maßnahme ausschlaggebend für den Einsatz der faktisch benachteiligenden Maßnahme war, sondern ein anderer Grund. Dies geschieht auf Ebene der

44 Dies deshalb, weil der Unterschied an der Konzeption des Verbots mittelbarer Diskriminierungen als Erleichterung zum Nachweis unmittelbarer Diskriminierungen insofern nichts ändert, als er notwendig aus dem äußeren Anknüpfen an ein anderes als das an sich verbotene Unterscheidungsmerkmal folgt. Im Übrigen ist auch bei unmittelbarer Diskriminierung letztlich zu fragen, ob das unzulässige Merkmal kausal für den Einsatz der z.B. Einzelmaßnahme wie etwa Kündigung war. 
„Rechtfertigungsprüfung “, wobei nach diesem Verständnis konsequenterweise keine hohen Anforderungen an die Rechtfertigung gestellt werden dürfen. Diese Anforderungen sind deshalb nicht hoch, weil es materiell gerade nicht um die Rechtfertigung einer Benachteiligung geht, die ohne Zweifel durch das unzulässige Unterscheidungsmerkmal verursacht wurde, sondern bloß um eine Widerlegung eines bloß vermuteten Kausalzusammenhanges. Für eine Rechtfertigungsprüfung im Sinne einer umfassenden Interessenabwägung nach den Kriterien der Sachlichkeit, Erforderlichkeit und Verhältnismäßigkeit ist bei dieser Sichtweise kein Platz.

Dem entspricht es, wenn der EuGH in den älteren Entscheidungen zur mittelbaren Diskriminierung keine besonders hohen Anforderungen an die Rechtfertigung stellt und bloß einen objektiv-sachlichen Grund verlangt, der nichts mit einer Diskriminierung auf Grund des unzulässigen Merkmals zu tun haben darf. ${ }^{45}$ Dies ist insofern konsequent, als es zur Widerlegung der Kausalitätsvermutung an sich nicht einmal eines sachlichen Grundes bedürfte. Theoretisch schließt jeder vom unzulässigen Merkmal verschiedene Grund für den Einsatz der benachteiligenden Maßnahme den vermuteten Kausalzusammenhang und damit auch die Vermutung einer Umgehung des Verbots der unmittelbaren Diskriminierung aus. Die Diskriminierungsverbote verlangen ja - jedenfalls von Privaten - nicht allgemein eine sachliche Entscheidung, sondern sie untersagen nur, die Entscheidung auf Grund unzulässiger Kriterien zu treffen. Selbst vollkommen subjektive Entscheidungskriterien Privater sind zulässig, sofern sie nur glaubhaft sind. ${ }^{46}$ Wenn der EuGH dennoch auch in den älteren Urteilen zur mittelbaren Diskriminierung zumindest einen objektiven und sachlichen (z.B. unternehmensspezifischen) Grund für den Einsatz der benachteiligenden Maßnahme verlangt, so dürfte dies wohl daran liegen, dass praktisch nur objektiv nachvollziehbare Motive ausreichend plausibel sind, um den vermuteten Kausalzusammenhang zu widerlegen.

Als Zwischenergebnis lässt sich daher festhalten, dass bei mittelbarer Diskriminierung nur insoweit von einem Kausalzusammenhang zwischen unzulässigem Merkmal und Benachteiligung gesprochen werden könnte, als man die tatsächlich nachteilige Auswirkung der Maßnahme als widerlegliche Vermutung für den deshalb un-

45 EuGH, Rs. 152/73, Sotgiu, Slg. 1974, 153; EuGH, Rs. 96/80, Jenkins, Slg. 1981, 911. In der E Jenkins hat es der EuGH als gerechtfertigt angesehen, wenn der Arbeitgeber aus wirtschaftlichen Gründen Teilzeitbeschäftigte benachteiligt, um einen Anreiz zur Vollbeschäftigung zu schaffen und auf diese Weise Personalreserven für Spitzenzeiten aufzubauen. Der EuGH spricht hier im Rahmen der Rechtfertigungsprüfung auch ausdrücklich von Gründen, die eine Diskriminierung auf Grund des Geschlechts ausschließen und stellt ausdrücklich auf die Beweggründe des Arbeitgebers für die Setzung einer mittelbar benachteiligenden Maßnahme ab, um festzustellen, ob nicht in Wahrheit eine Diskriminierung auf Grund des Geschlechts vorliegt. Diese Formulierungen deuten ebenfalls in Richtung eines Verständnisses der mittelbaren Diskriminierung als Beweislastregel zur Feststellung unmittelbarer Diskriminierungen.

46 Treffend wird daher gesagt, dass die Gleichbehandlungs-Richtlinien es dem Arbeitgeber nicht verbieten, das Nichteinstellen mit fehlender Sympathie zu begründen - vorausgesetzt dies ist glaubhaft. 
terstellten Kausalzusammenhang ansieht. In diesem Fall ist die im Schrifttum umstrittene Frage, ob die Rechtfertigungsmöglichkeit der mittelbaren Benachteiligung ein Element der Kausalitätsprüfung ist, eindeutig zu bejahen. Dem Verbot mittelbarer Diskriminierung kommt dann allerdings kein eigenständiger Gerechtigkeitsgehalt zu, weil es bloß als Erleichterung zum Nachweis (verdeckter) unmittelbarer Diskriminierung dient. Diese Auffassung dürfte auch heute noch weit verbreitet sein. ${ }^{47}$

\section{Entwicklung zum „kausalitätsfreien“ Verbot mittelbarer Diskriminierung}

Die Auffassung, das Verbot mittelbarer Diskriminierung sei nur eine Absicherung des Verbotes - notwendig kausaler - unmittelbarer Diskriminierung, findet in der neueren Judikatur des EuGH aber keine Stütze mehr.

\section{Verschärfung der Rechtfertigungsprüfung}

Zum Gleichbehandlungsrecht hat der EuGH bereits 1986 in der Entscheidung Bil$k a$ die Anforderungen an die Rechtfertigung deutlich verschärft und für den Ausschluss von Teilzeitbeschäftigten aus einem betrieblichen Ruhegeldsystem verlangt, dass diese Maßnahme einem wirklichen Bedürfnis des Unternehmens dienen und für die Erreichung dieses Ziels geeignet und erforderlich sein muss. ${ }^{48}$ Diese Formel wiederholt der EuGH seither in ständiger Rechtsprechung und setzt damit zur mittelbaren Diskriminierung nunmehr einen strengen Prüfungsmaßstab, der deutlich über eine bloße Sachlichkeits- und Plausibilitätsprüfung hinausgeht. ${ }^{49}$ Gefordert wird eine echte Verhältnismäßigkeitsprüfung im Sinne einer umfassenden Abwägung zwischen Gleichberechtigungszielen und Zweck der in Frage stehenden Regelung. Damit geht die Funktion des Verbots mittelbarer Diskriminierungen aber weit über ihre ursprüngliche Funktion als Beweiserleichterung hinaus, wenn und weil für die Widerlegung einer Kausalitätsvermutung bereits ein schlichter Sachgrund ausreicht.

Illustrative Beispiele bieten auch die Urteile Bötel und Lewark, in welchen der EuGH eine nationale Regelung zur Entgeltfortzahlung während Betriebsratstätigkeit als mittelbare Geschlechtsdiskriminierung verworfen hat. ${ }^{50} \mathrm{Um}$ dem Charakter der Betriebsratstätigkeit als Ehrenamt gerecht zu werden, sah die Regelung nur die Fortzahlung tatsächlich entfallener Arbeitsstunden vor. Der EuGH erblickte darin aber eine rechtfertigungsbedürftige mittelbare Benachteiligung von Teilzeitbeschäftigen und damit von Frauen, wenn Teilzeitbeschäftigte nach der nationalen Norm in ab-

47 Z.B. G. Thüsing, Arbeitsrechtlicher Diskriminierungsschutz, München 2007, S. 103; ders., Das Arbeitsrecht der Zukunft? NZA-Beilage zu Heft 22/2004, S. 3 (6); Plötscher, Diskriminierungsbegriff (Fn. 17, S. 293; Schlachter, Diskriminierung (Fn. 17), S. 190; in diese Richtung auch Temming, Altersdiskriminierung (Fn. 1), S. 440, wonach die Figur der mittelbaren Diskriminierung der Absicherung des Verbots unmittelbarer Diskriminierungen dient und die Funktion einer Beweislastregel hat.

48 EuGH, Rs. 170/84, Bilka, Slg. 1996, 1607.

49 Vgl. nur EuGH, Rs. C-171/88, Rinner-Kühn, Slg. 1989, I-02743; Rs. C-226/98, Joergensen, Slg. 2000, I-02447; Rs. C-381/99, Brunnhofer, Slg. 2001, I-04961.

50 EuGH, Rs. C-360/90, Bötel, Slg. I-03589; Rs. C-457/93, Lewark, Slg. 1996, I-00243. 
soluten Zahlen einen geringeren Erstattungsbetrag für die Betriebsrats-Tätigkeit erhielten als Vollzeitbeschäftigte. Unabhängig davon, wie man zu dieser Entscheidung inhaltlich steht, geht sie im Ergebnis deutlich über ein Diskriminierungsverbot wegen des Geschlechts oder eines anderen unzulässigen Merkmals hinaus. Ein entsprechender Kausalzusammenhang ist wohl bereits wegen der Ehrenamtlichkeit der Betriebsratsfunktion ausgeschlossen.

Das Verbot mittelbarer Diskriminierungen verfolgt damit heute eigenständige Gleichstellungsziele, die auf Beseitigung tatsächlich bestehender Nachteile bestimmter Personengruppen abzielen und damit über die den unmittelbaren Diskriminierungsverboten immanenten Anforderungen nach individueller Gerechtigkeit hinausgehen. ${ }^{51}$ Es kommt wegen der hohen Anforderung an die Rechtfertigung heute daher im Ergebnis weniger auf Motiv oder Ursache für den Einsatz einer mittelbar benachteiligenden Regelung an, sondern primär auf ihre tatsächliche oder potentielle Auswirkung. ${ }^{52}$ Die hohe Rechtfertigungslast bei Verwendung eines an sich neutralen Differenzierungsmerkmals nähert das Verbot mittelbarer Diskriminierung im Rahmen der Gleichbehandlung einem allgemeinen Gleichheitssatz an, weil es im Ergebnis immer weniger auf die Anknüpfung an ein bestimmtes (verbotenes) Differenzierungsmerkmal ankommt. ${ }^{53}$

Auf einen Kausalzusammenhang zwischen unzulässigem Merkmal und benachteiligender Maßnahme kommt es daher (zumindest heute) nicht mehr an, wenn und weil nur noch gefragt wird, ob die nachteiligen Auswirkungen der Maßnahme als solche einer Verhältnismäßigkeitsprüfung standhalten oder nicht. Maßgeblich ist nicht, ob das neutrale Unterscheidungskriterium wegen einer typischerweise nachteiligen Wirkung auf die geschützte Personengruppe gewählt wurde, sondern nur noch die nachteilige Wirkung der (an das neutrale Kriterium anknüpfenden) Maßnahme selbst.

Bei einer solch wirkungsorientierten Betrachtungsweise ergibt sich der Vorwurf der mittelbaren Diskriminierung dann freilich immer weniger aus der beanstandeten

51 In diese Richtung z.B. auch Adomeit/Mohr, AGG (Fn. 21), S. 274 ff.; D. Schiek, Kalanke und die Folgen - Überlegungen zu EG-rechtlichen Anforderungen an betriebliche Gleichstellungspolitik, Arbeit und Recht 1996, S. 128 (130).

52 Heun (Fn. 1), Art. 3 Rn. 108, 124 bezeichnt die so verstande mittelbare Diskriminierung daher insofern zu Recht als faktische Diskriminierung, die als bloße Beweiserleichterung verstandene hingegen als verdeckte bzw. versteckte Diskriminierung. Da die Begriffe faktische, verdeckte und versteckte Diskriminierung aber in Schrifttum und Judikatur nicht einheitlich, sondern mit unterschiedlichem Sinngehalt verwendet werden, empfielt es sich, aus Gründen der klaren Begriffsbildung jedes (unzulässige, weil nicht rechtfertigbare) Anknüpfen an ein neutrales (aber faktisch benachteiligendes) Kriterium als mittelbare Diskriminierung zu bezeichnen, deren Verbot heute eben andere Funktionen erfüllt als früher.

53 Treffend Plötscher, Diskriminierungsbegriff (Fn. 17), S. 55. Exemplarisch für dieses Phänomen auch der Schlussantrag von GA Stix-Hackel zu Rs. C-400/02, Merida, Slg. 2004, I-8471, Rn. 20, wo sie nicht bloß die Anwendung unterschiedlicher Vorschriften auf gleiche Sachverhalte als mittelbare Diskriminierung ansieht, sondern auch die Anwendung derselben (!) Vorschrift auf unterschiedliche Sachverhalte und damit den Unterschied zum allgemeinen Gleichheitssatz vollends auflöst. 
Reglung selbst, sondern immer mehr aus den faktischen Verhältnissen, auf welche die Regelung trifft. Auch unter diesem Gesichtspunkt ist dann für das Erfordernis eines Kausalzusammenhanges zwischen spezifisch unzulässigem Merkmal und Benachteiligung kaum Platz.

Diese Lockerung der Anforderungen an einen Zusammenhang mit dem unzulässigen Unterscheidungsmerkmal zeigt sich auch bei der Umschreibung der faktischen Nachteiligkeit einer beanstandeten Regelung im Sekundärrecht. Schon die „alte“ Definition der Beweislast-RL aus 1996 zur mittelbaren Diskriminierung verlangte nur, dass die beanstandete Regelung tatsächlich überwiegend Personen mit bestimmter Ausprägung des unzulässigen Merkmals trifft. Es genügte also eine deutliche Korrelation zwischen Benachteiligung und unzulässigem Kriterium, wie sie in den erwähnten Fällen besteht: Personen fremder ethnischer Herkunft sprechen die Landessprache üblicherweise nicht so gut wie Einheimische; Frauen betreuen Kinder weitaus häufiger als Männer und arbeiten weitaus häufiger Teilzeit als Männer. Die „neue“ Definition geht weiter und verlangt nur noch die besondere Eignung der Regelung zur Benachteiligung. Dies bedeutet nicht bloß, dass die mittelbare Benachteiligung nunmehr auch ohne statistischen Nachweis einer tatsächlichen Benachteiligung bejaht werden kann. Es bedeutet zugleich eine Ausweitung des äußerst möglichen Vergleichsrahmens zur Feststellung der Benachteiligung, wenn und weil die abstrakte Eignung einer Regelung zur Benachteiligung ausreicht. Die modifizierte Formulierung in der neuesten RL 2006/54/EG könnte dies noch etwas erweitern, indem sie nur mehr eine „Situation“ verlangt, in der dem Anschein nach neutrale Kriterien eine bestimmte Gruppe besonders benachteiligen können.

$\mathrm{Zu}$ erwägen ist, ob die hohe Rechtfertigungslast einschließlich Verhältnismäßigkeitsprüfung dahingehend verstanden werden kann, dass (unabhängig von der Ursächlichkeit des unzulässigen Merkmals) sichergestellt werden soll, dass der vorgebrachte Rechtfertigungsgrund wesentliche Bedingung für den Einsatz einer faktisch benachteiligenden Regelung ist. Für diese Frage kommt es nicht darauf an, ob das unzulässige Merkmal ursächlich war oder nicht, sondern nur darauf, ob allein der vorgebrachte Grund für die Maßnahme wertungsmäßig so überzeugend ist, dass er (auch im Vergleich mit anderen Ursachen) jedenfalls als die wesentliche Ursache anzusehen ist. ${ }^{54}$ Auch diese Sichtweise ändert aber nichts daran, sondern bestätigt, dass das Vorliegen oder Nichtvorliegen einer mittelbaren Diskriminierung ohne Rücksicht auf einen Kausalzusammenhang zwischen benachteiligender Maßnahme und verbotenem Unterscheidungsmerkmal zu beurteilen ist. Die Frage nach der „Wesentlichkeit" des neutralen Kriteriums erfordert letztlich ähnliche Wertungen wie jene nach der Rechtfertigung der besonderen Beeinträchtigung. 
Das Abgehen vom Kausalitätserfordernis ist im Arbeitsrecht insbesondere für Maßnahmen des einzelnen Arbeitgebers relevant. Während bei diesen nach „alter“ Definition für die Feststellung der Benachteiligung der Vergleichsrahmen auf die Arbeitnehmer eines Betriebes oder Unternehmens beschränkt war, kann es heute für die Beurteilung der objektiven Eignung einer Regelung zur Benachteiligung nicht mehr (nur) auf die tatsächlichen Verhältnisse beim beklagten Arbeitgeber ankommen, sondern (auch) auf die gesamtwirtschaftlichen Umstände. ${ }^{55}$ Differenziert der Arbeitgeber z.B. nach dem Beschäftigungsausmaß, so kann dies auch dann mittelbare Diskriminierung nach dem Geschlecht sein, wenn im Betrieb der Anteil der Männer an den Teilzeitbeschäftigten höher ist. Ein solch abstraktes Verständnis der mittelbaren Benachteiligung kann wohl nicht einmal mehr als Vermutung gedeutet werden, dass die konkrete Benachteiligung durch das Geschlecht verursacht wird. Allerdings könnte man erwägen, dass die Benachteiligung von Teilzeitbeschäftigten zumindest auf dem gesamten Arbeitsmarkt durch das Geschlecht verursacht wird. Auch auf dieser Ebene fehlt es aber an einer conditio oder einer gesetzmäßigen Bedingung im Sinne der traditionellen Kausalitätstheorien.

Es lässt sich daher festhalten, dass es im Recht der Gleichbehandlung nach heutigem Verständnis von Zweck und Funktion des Rechtsinstituts der mittelbaren Diskriminierung für einen Diskriminierungsvorwurf nicht auf einen Kausalzusammenhang zwischen unzulässigem Merkmal und Benachteiligung in der Weise ankommt, dass das unzulässige Merkmal bzw. die darauf bezogene nachteilige Wirkung einer beanstandete Maßnahme Grund für den Einsatz der Maßnahme ist. Daher kann auch die Frage der Rechtfertigung keine Frage der Kausalität sein, sondern nur eine Frage der Interessenabwägung zwischen Maßnahmenzweck einerseits und ihren nachteiligen Auswirkungen im Hinblick auf Gleichstellungsinteressen andererseits. ${ }^{56}$

55 Die Rechtsprechung des EuGH zu dieser Frage ist uneinheitlich, weil der Gerichtshof manchmal faktische Wirkungen in den Vordergrund rückt (z.B. Rs. 170/84, Bilka, Slg. 1986, 1607; Rs. 171/88, Rinner-Kühn, Slg. 1989, 2743; Rs. C-266/95, Merino, Slg. 1997, I-3279), das andere Mal - insbes. zu Freizügigkeiten - hingegen die potentielle Eignung (z.B. Rs. C-204/90, Bachmann, Slg. 1992, I-129; Rs. C-317/93, Nolte, Slg. 1995, I-04625; Rs. C-237/94, O Flynn, Slg. 1996, I-2617).

56 Oben wurde bereits dargelegt, dass die Frage, ob bei Rechtfertigung einer mittelbaren Benachteiligung die Kausalität entfalle oder nicht, für sich genommen keine sehr große Bedeutung hat. Tobler, Indirect Discrimination (Fn. 17), S. 254, nennt aber zwei Punkte, die doch gegen die Zuordnung zur Kausalität sprechen sollen. (1) Der im Rahmen der Interessenbeeinträchtigungsprüfung vorzunehmende Abwägungsprozess zwischen Gleichberechtigungszielen und Zielen der zu beurteilenden Maßnahme sei im Rahmen einer Kausalitätsprüfung kaum möglich. Dies trifft zu, wenn man die „gereinigten“ Kausalitätskonzepte zugrunde legt. Daneben werden aber noch immer Kausalitätskonzepte mit wertenden Betrachtungen und Beschränkungen verwendet (vgl. oben Fn. 23), und das Unionsrecht hat bislang kein eigenes Kausalitätskonzept entwickelt. Kausalitätsprüfung und Abwägungsprozesse schließen einander daher nicht notwendig aus. Ferner könne nach Tobler (2) eine Prüfung der Rechtfertigung auf Kausalitätsebene die Wirkungsweise des Andidiskriminierungsrechts insofern beeinträchtigen, als dann eine gerechtfertigte mittelbare Benachteiligung erst gar nicht als Benachteiligung wegen eines unzulässigen Unterscheidungsmerkmals anzusehen ist. Dieser Einwand betrifft eher die psychologischen Auswirkungen der Einordnungsfrage. Danach spricht für die Einordnung als echter Rechtfertigungsgrund, dass dann aus Sicht der Betroffenen trotz gerechtfertigter Benachteiligung klar ist, dass 


\section{Kausalzusammenhang zwischen unzulässigem Merkmal und Korrelation?}

Sucht man vor diesem Hintergrund dennoch nach für den Tatbestand der mittelbaren Diskriminierung relevanten Kausalzusammenhängen zwischen unzulässigem Merkmal und Benachteiligung, so könnte man noch fragen, ob die überproportional nachteiligen Auswirkungen der beanstandeten Regelung auf Personen mit spezifischer Ausprägung des unzulässigen Merkmals durch Eigenheiten eben dieses Merkmals verursacht sein müssen. Allein aus der spezifisch nachteiligen Auswirkung der Regelung in eine Richtung folgt ein solcher Kausalzusammenhang wie gesagt noch nicht. Die wirkungsorientierte Betrachtung einer Reglung sagt ja nur, dass das verwendete, dem Anschein nach neutrale Kriterium (und damit auch die nachteilige Wirkung der danach differenzierenden Regelung) mit dem unzulässigem Merkmal stark korreliert (z.B. Teilzeitbeschäftigung und weibliches Geschlecht), dass also die nachteilige Wirkung eines neutralen Unterscheidungsmerkmals je nach Ausprägung des unzulässigem Merkmals ungleich verteilt ist (z.B. zwischen männlichen und weiblichen Personen beim Merkmal Geschlecht).

Es kann dann nur noch fraglich sein, ob schon diese Korrelation im Sinne einer überwiegenden Betroffenheit allein ausreicht, um für den Beklagten die Rechtfertigungspflicht auszulösen, oder ob zusätzlich feststehen muss, dass diese Korrelation gerade wegen des unzulässigen Merkmals auftritt. Dabei wird anders als bei der bisher behandelten Frage nicht gefragt, ob das unzulässige Merkmal kausal für die Benachteiligung (im Einzelfall) ist, sondern danach, ob das unzulässige Merkmal kausal für die ungleiche Verteilung der nachteiligen Auswirkungen je nach Ausprägung des unzulässigen Merkmals und damit kausal für den aus Sicht des Gleichbehandlungsrechts unerwünschten Zustand ist.

Die Erforderlichkeit einer solchen zur Korrelationsprüfung zusätzlichen Kausalitätsprüfung war früher umstritten, wird heute aber überwiegend abgelehnt. ${ }^{57}$ Allerdings betrifft diese Frage letztlich die Aussagekraft der im Verfahren vorgelegten statistischen Daten. Im Gleichbehandlungsrecht hat sie sich in der Praxis bisher wohl deshalb nur selten gestellt, weil der entsprechende Kausalzusammenhang meist offenkundig war. Dies betrifft insbesondere die Urteile zu Differenzierungen nach dem Beschäftigungsausmaß, wo die überwiegend nachteilige Betroffenheit von Frauen leicht mit geschlechtsspezifischen Gründen erklärt werden konnte; nämlich dadurch, dass die historisch gewachsene Verteilung der Geschlechterrollen innerhalb der Fa-

sie eigentlich (wenn auch gerechtfertigt) „diskriminiert“ wurden. Diese einseitige Sicht ist aber wenig überzeugend, weil man ebenso - entgegengesetzt - aus Sicht des Handelnden argumentieren könnte, der dem Diskriminierungsvorwurf ausgesetzt ist.

57 Vgl. nur R. Rebhahn, in: R. Rebhahn (Hrsg.), Gleichbehandlungsgesetz-Kommentar, Wien: Springer 2005 , 5 Rn. 38. 
milie es verheirateten Frauen außerordentlich schwer macht, eine vollberufliche Erwerbstätigkeit mit ihren familiären Belastungen zu verbinden. ${ }^{58}$

Man sollte diese zusätzliche Kausalitätsprüfung grundsätzlich zulassen, schon um das Wesen des Verbots mittelbarer Diskriminierung als besonderes (in Bezug auf ein spezifisches Merkmal bestehendes) Diskriminierungsverbot zu bewahren und um statistische Zufälligkeiten auszuschließen. So erscheint es z.B. im Sachverhalt des „wegweisenden“ Urteils Enderby ${ }^{59}$ durchaus fraglich, ob der Logopädenberuf (anders als der Apotheker- oder Psychologenberuf) aus geschlechtsspezifischen Gründen überwiegend von Frauen ausgeübt wird. Man sollte daher wohl auch die dort statistisch belegte überproportionale Benachteiligung beim Entgelt bloß als widerlegbare Vermutung für einen Ursachenzusammenhang zwischen unzulässigem Merkmal und Korrelation ansehen. ${ }^{60}$

\section{Umgekehrter Kausalverlauf?}

Bisher ging es stets um die Frage, ob das unzulässige Merkmal Ursache der nachteiligen Rechtsfolge bzw. der besonderen Betroffenheit ist (was wie gesagt weder erforderlich ist noch zutrifft). Die Frage, ob das neutrale Kriterium bestimmte Personen im Lichte des unzulässigen Merkmals „in besonderer Weise benachteiligen kann“, scheint aber auch andere Fragen zur Kausalität aufzuwerfen. Das Konzept der Eignung zur bzw. der Gefahr einer Benachteiligung scheint wesentlich auf die Kategorie der Wahrscheinlichkeit und damit auf mögliche Kausalverläufe zu rekurrieren. Allerdings handelt es sich dabei wenn, dann um den umgekehrten Kausalverlauf wie jenem, nach dem bisher gefragt wurde. Nun geht es nicht darum, ob das unzulässige Merkmal kausal für die (nachteilige) Rechtsfolge ist, sondern um die Frage, ob und inwieweit die neutrale Vorschrift bzw. Maßnahme Ursache für die „,besondere Benachteiligung “ ist. Diese Frage scheint insbesondere bei den vier Freiheiten relevant sein.

Als Beispiel sei wieder das nationale Verbot des Verkaufs von Medikamenten über das Internet gewählt. Dazu ist zu fragen, ob „eine Situation“ vorliegt, „in der dem Anschein nach neutrale Vorschriften, Kriterien oder Verfahren“ ausländische An-

58 Vgl. z.B. EuGH, Rs. 170/84, Bilka, Slg. 1986, 1607. Anders ist es aber bei der Besetzung von Führungspositionen durch einen bestimmten Arbeitgeber. Dazu erging im Juli 2010 ein Urteil des BAG (GEMA-Fall, 8 AZR 1012/08), das aber noch nicht veröffentlicht ist.

59 EuGH, Rs. C-127/95, Enderby, Slg. 1993, I-05535.

60 Für diese Auffassung spricht wohl auch die bereits erwähnte Neufassung der RL-Definition des Begriffes der mittelbaren Diskriminierung, wonach es nicht mehr auf überwiegende tatsächliche Betroffenheit ankommen soll, sondern auf die spezifische Eignung einer Regelung zur spezifischen Benachteiligung von Personen mit der Ausprägung des unzulässigem Merkmals. Die Frage der Eignung zur Benachteiligung lässt sich ohne Rücksicht auf die tatsächliche Betroffenheit wohl nur bejahen, wenn das neutrale Kriterium von Personengruppen, die nach dem unzulässigen Merkmal gebildet werden, typischerweise leichter oder schwieriger erfüllt werden kann (z.B. Körperkraft im Hinblick auf das Geschlecht). 
bieter „in besonderer Weise gegenüber“ inländischen Anbietern „benachteiligen können". Der EuGH hat dies bejaht. ${ }^{61}$ Legt man hier den conditio-Test an und denkt sich das Internetverkaufsverbot weg, so bessert sich die Lage nicht nur für ausländische, sondern auch für fast alle inländischen Apotheken, mag die Zahl der ausländischen betroffenen Apotheken auch deutlich höher sein. Die hohe Korrelation der geprüften Rechtsfolge mit dem unzulässigen Merkmal begründet auch hier keinen Kausalzusammenhang. Richtigerweise geht es auch bei der „besonderen Benachteiligung “ nicht um einen Kausalzusammenhang, sondern (nur) um Wahrscheinlichkeit und Betroffenheit.

Diese Frage nach der Wahrscheinlichkeit einer im Hinblick auf das unzulässige Merkmal „besonderen Auswirkung“ wirft nun bei den vier Freiheiten aus strukturellen Gründen besondere Schwierigkeiten auf, auf die kurz eingegangen sei, auch wenn sie über Kausalitätsfragen hinausgreifen. Die in den Gleichbehandlungs-Richtlinien „neue“ Definition geht zwar auf ältere Judikatur des EuGH zu den Freizügigkeiten zurück. Die Ablösung des Diskriminierungsverbotes vom Kausalitätserfordernis (zwischen unzulässigem Merkmal und Rechtsfolge) führt bei den Freizügigkeiten nun nicht zu einer steten Annäherung an einen allgemeinen Gleichheitssatz, sondern zu einer Annäherung an die Grundrechte (insbesondere auf Berufsfreiheit), die sich insbesondere in der Erweiterung zu Beschränkungsverboten zeigt.

Die erwähnten Schwierigkeiten resultieren zum Teil daraus, dass sich eine nationale Regelung aus strukturellen Gründen hier weit eher auf Träger des unzulässigen Merkmals (z.B. ausländische Verkäufer) nachteilig auswirkt als sich eine Maßnahme im Bereich der Gleichbehandlungsvorschriften ungleich in Bezug auf das unzulässige Merkmal auswirkt. Fast alle Diskriminierungsverbote der Gleichbehandlungsvorschriften wirken nämlich zwei- oder allseitig (unzulässig ist die Diskriminierung z.B. aufgrund des Alters, nicht nur die der Älteren). Bei den Freizügigkeiten gibt es hingegen nur eine Gruppe, deren Schutz das Diskriminierungsverbot dient (Personen, die den Sachverhalt von einem anderen Mitgliedstaat aus verwirklichen wollen).

Bei den vier Freizügigkeiten führt das unzulässige Merkmal wie gesagt nicht selten notwendig dazu, dass die geprüfte Rechtsfolge eingreift, auch wenn es nicht Ursache i.e.S. für das Eingreifen ist. Man könnte nun erwägen, dass sich eine Maßnahme wie das erwähnte Verkaufsverbot deshalb „besonders“ auf die Unternehmer aus anderen Mitgliedstaaten auswirkt, weil es dort auch real (Internetverkauf) stets mehr Anbieter als im verbietenden Mitgliedstaat geben kann und geben wird. Allerdings würde dieses Verständnis die Worte „in besonderer Weise“ hier ihres Sinnes berauben. Denn in der EU-27 gibt es in anderen Mitgliedstaaten fast stets mehr Anbieter als im regelnden Mitgliedstaat. Eine andere Begründung für ein Auswirken „in besonderer

61 EuGH, Rs. C-322/01, Dt. Apotherkerverband, Slg. 2003 I-14887 Rn.74. Kritisch Kingreen, Grundfreiheiten (Fn. 15), S. 729. 
Weise“ ist im konkreten Fall aber nicht ersichtlich. ${ }^{62}$ Das fragliche Verkaufsverbot ist daher keine mittelbare Diskriminierung. ${ }^{63}$ Man kann das fragliche Verbot mit Artikel 34 AEUV nur bekämpfen, wenn man ihm auch ein Beschränkungsverbot entnimmt, und damit ein Grundrecht auf Berufsfreiheit. ${ }^{64}$ Die hohe Korrelation des unzulässigen Merkmals mit der geprüften Rechtsfolge sagt in diesem Fall - anders als bei den Gleichbehandlungsvorschriften - nämlich inhaltlich kaum etwas über die Regelung selbst aus.

\section{E. Schlussbemerkung}

Bei Verboten der unmittelbaren Diskriminierung ist es klar, dass diese nur vorliegt, wenn aufgrund des unzulässigen Unterscheidungsmerkmals verschieden behandelt wird, wenn also dieses Merkmal Ursache - verstanden im Sinne der traditionellen Konzepte zur Kausalität im Recht - für die unterschiedliche Behandlung bzw. Rechtsfolge ist.

Auch zu den Verboten mittelbaren Diskriminierung des aktuellen Unionsrechts (insbesondere Gleichbehandlung, Freizügigkeiten) wird weithin davon ausgegangen, dass das unzulässige Merkmal „Ursache“ einer von diesen Verboten erfassten unterschiedlichen Behandlung bzw. einer Diskriminierung ist. Diskutiert wird allenfalls darüber, ob bei Rechtfertigung der unterschiedlichen Behandlung nur das Verbot oder auch die Kausalität des Merkmals für die Benachteiligung entfällt. Nähere Betrachtung zeigt aber, dass das heutige Verständnis des Verbotes mittelbarer Diskriminierung eine Kausalität zwischen unzulässigem Merkmal und verbotenem „Verhalten" nicht erfordert, ja mit dem Erfordernis einer solchen Kausalität gar nicht vereinbar wäre. Dieses Kausalitätserfordernis lag zwar nahe, solange das Verbot mittelbarer Diskriminierung nur als Annex zum Verbot unmittelbarer Diskriminierung verstanden wurde; man behalf sich mit der Vermutung einer - kausalen - unmittelbaren Diskriminierung. Seither wurde das Verbot mittelbarer Diskriminierung aber - zur Gleichbehandlung auch vom EU-Gesetzgeber - zu einem von der fraglichen Kausalität losgelösten Verbot fortentwickelt.

62 Auch das Urteil des EuGH sagt dazu nichts. Vielmehr erweckt es in der entscheidenden Rn. 74 den Eindruck, als wäre jede deutsche Apotheke im ganzen Bundesgebiet präsent bzw. als gäbe es nur eine einzige Apotheke in Deutschland.

63 Es ist daher auch missverständlich, wenn eine unterschiedslos anwendbare Regelung bloß wegen ihrer unterschiedlichen Auswirkung mitunter als „materiell diskriminierend“ bezeichnet wird.

64 Würde man hingegen „in besonderer Weise“ so weit verstehen wie eben angedeutet, dann bringt eine Reduktion der Freizügigkeiten auf Diskriminierungsverbote keine Änderung gegenüber dem Beschränkungsverbot, sondern eher nur eine Reduktion der Argumentation. Ähnliches gilt auch in Bezug auf die nationale Beschränkung des Aufbackens tief gefrorener Backwaren auf Personen mit Gewerbebefugnis für das volle Bäckereigewerbe. Auch hier kann man nur dann sagen, dass diese Regelung ausländische Anbieter „,in besonderer Weise gegenüber“ inländischen Anbietern „benachteiligen könnte“, wenn man annimmt dass, dass ausländische Produzenten beim Verkauf in einen anderen MS mehr auf Tiefgefrorenes setzen müssten, und dies dann ausreichen lässt. So aber SA GA Poiares Maduro zu EuGH, verb.Rs. C-158/04 und C-159/04, Alfa Vita Vassilopoulos, Slg. 2006, I-8153 Rn. 21. 
Gleichwohl spielen Fragen, die im Bereich der Kausalität angesiedelt sind, auch bei der mittelbaren Diskriminierung eine Rolle. So wären in der „Dogmatik“ der Freizügigkeiten vielleicht jene Fälle gesondert zu erfassen, bei denen die Verwirklichung des unzulässigen Merkmals die geprüfte Rechtsfolge notwendig nach sich zieht. 